\title{
ВОЗМОЖНОСТИ ПРИМЕНЕНИЯ ЭКЗОСОМ ДЛЯ ДИАГНОСТИКИ И ЛЕЧЕНИЯ МИОДИСТРОФИИ ДЮШЕННА
}

\section{И. И. Галкин ${ }^{1,2}$, Т. В. Егорова ${ }^{1,3} \bowtie$}

1 ООО «Марлин Биотех», Москва, Россия

2 Институт физико-химической биологии имени А. Н. Белозерского, Москва, Россия

${ }^{3}$ Институт биологии гена, Москва, Россия

\begin{abstract}
Миодистрофия Дюшенна - наиболее часто встречающаяся форма миодистрофии. Однако эффективного лечения этого заболевания до сих пор не разработано. В настоящее время появляются работы, посвященные использованию экзосом в моделях миодистрофии Дюшенна. Экзосомы, небольшие (40-100 нм) везикулы, секретируемые клетками в межклеточное пространство, переносят некоторые макромолекулы (микроРНК, белки), анализ которых может быть использован для неинвазивной оценки успешности применяемой терапии. Кроме того, они могут обеспечивать адресную доставку активных веществ (например, микроРНК, антисмысловых олигонуклеотидов). Анализируя имеющиеся данные и оценивая возможность диагностики и терапии дистрофии Дюшенна с помощью этих везикул, мы предполагаем, что экзосомы могут занять место в арсенале исследователей и врачей, если удастся решить некоторые технические проблемы.
\end{abstract}

Ключевые слова: миодистрофия Дюшенна, экзосомы, адресная доставка, жидкостная биопсия, генетическая терапия

Информация о вкладе авторов: И. И. Галкин, Т. В. Егорова - анализ литературы, написание текста статьи.

$\triangle$ Для корреспонденции: Татьяна Владимировна Егорова

ул. Вавилова, 34/5, г. Москва, 119334; t.dimitrieva@marlinbiotech.com

Статья получена: 21.07.2019 Статья принята к печати: 05.08.2019 Опубликована онлайн: 12.08.2019

DOI: $10.24075 /$ vrgmu.2019.049

\section{THE POTENTIAL OF EXOSOMES FOR THE DIAGNOSIS AND TREATMENT OF DUCHENNE MUSCULAR DYSTROPHY}

\section{Galkin $\|^{1,2}$, Egorova TV1,3凶}

${ }^{1}$ Marlin Biotech LLC, Moscow, Russia

${ }^{2}$ A. N. Belozersky Institute of Physico-Chemical Biology, Moscow, Russia

${ }^{3}$ Institute of Gene Biology, Russian Academy of Sciences, Moscow, Russia

\begin{abstract}
Duchenne muscular dystrophy is the most common type of muscular dystrophy. There is no effective cure for this disease. Recently, researchers have started to look at the therapeutic potential of exosomes - small $(40-100 \mathrm{~nm})$ vesicles secreted by cells into the extracellular environment. They transport a few types of macromolecules, including microRNA and proteins, that can be analyzed to estimate the efficacy of the applied therapy. Besides, exosomes can be harnessed for delivering therapeutic components (microRNA, antisense oligonucleotides) to the target tissue. Below, we analyze the available literature and assess the feasibility of using exosomes in the diagnosis and treatment of Duchenne muscular dystrophy. We conclude that exosomes can have their place in the arsenal of researchers and clinicians once some technical issues are solved.
\end{abstract}

Keywords: Duchenne muscular dystrophy, exosomes, targeted drug delivery, liquid biopsy, gene therapy

Author contribution: Galkin II, Egorova TV — literature analysis and manuscript preparation.

$\triangle$ Correspondence should be addressed: Tatiana V. Egorova

Vavilova 34/5, Moscow, 119334; t.dimitrieva@marlinbiotech.com

Received: 21.07.2019 Accepted: 05.08.2019 Published online: 12.08.2019

DOI: 10.24075/brsmu.2019.049

Миодистрофия Дюшенна (МДД) - Х-сцепленное рецессивное заболевание, вызываемое мутациями в гене DMD. Мутации приводят к недостаточной выработке белка дистрофина либо к синтезу его нефункциональной формы. Болезнь проявляется в постепенной деградации мышечной ткани и развитии фиброза. В возрасте 8-12 лет пациент теряет способность двигаться самостоятельно, смерть чаще всего наступает от дыхательной или сердечной недостаточности в возрасте около 20 лет. При незначительной потере функциональности дистрофина развивается более мягкая форма - дистрофия Беккера.

Большинство применяемых на сегодняшний день методов лечения можно отнести к симптоматическим. Среди них - поддерживающая физкультура, легкие ортопедические вмешательства для предотвращения контрактур, респираторная поддержка, назначение глюкокортикоидов [1]. Эти меры позволяют продлить как период самостоятельной двигательной активности, так и продолжительность жизни (до 30 лет). Очевидно, данные подходы не направлены на устранение основной причины возникновения заболевания - недостатка или отсутствия функционирующего дистрофина. Восполнить недостаток этого белка можно с помощью разрабатываемых методов генной терапии. Основные подходы - доставка укороченного гена дистрофина (микродистрофина) с помощью аденоассоциированных вирусов (AAV) [2], модификация процессинга пре-мРНК (пропуск экзонов, или экзон-СКиппинг) с помощью модифицированных олигонуклеотидов [3] или системы CRISPR-Cas. Подход, связанный с доставкой гена с помощью AAV, ограничен из-за возникновения иммунного ответа на белки капсида, а небольшая емкость капсида вынуждает использовать укороченные формы дистрофина, уступающие по функциональности полноразмерному белку. Методики, основанные на пропуске экзонов, демонстрируют низкую функциональную эффективность. К тому же их применение ограничено конкретными мутациями, поэтому каждое лекарственное средство может быть 
применено для лечения только небольшой доли пациентов [3]. Общей проблемой, снижающей эффективность как существующих способов лечения МДД, так и перспективных методов генной терапии, является затруднение доставки действующего вещества в целевую ткань. Таким образом, и существующие, и перспективные методы терапии МДД имеют ряд серьезных ограничений и недостатков, и разработка новых подходов представляется крайне обнадеживающей. В последнее время появляется больше исследований, посвященных экзосомам, в том числе как потенциальным переносчикам действующих веществ [4].

Экзосомы - это класс внеклеточных везикул, секретируемых клетками в межклеточное пространство: кровь, лимфу, среду роста и т. д. Среди всех внеклеточных везикул экзосомы - наименьшие; в большинстве источников их размер определяют как 40-100 нм, реже 150 нм. В отличие от других внеклеточных везикул апоптотических телец (диаметр 50-1000 нм) и микровезикул, отшнуровывающихся от клеточной мембраны (диаметр 100-300 нм), экзосомы секретируются после слияния мультивезикулярного тела с мембраной [5]. Первоначально экзосомы считали своего рода «мусорными мешками", задача которых состоит в удалении ненужных веществ из клетки. На сегодняшний день продемонстрирована роль экзосом в переносе информации (преимущественно в виде белков и микроРНК) между клетками [5]. $\mathrm{Ha}$ липидной поверхности экзосом экспонированы некоторые белки группы кластеров дифференцировки (CD9, CD63 и др.), обнаружены компоненты главного комплекса гистосовместимости. Поверхностные белки предотвращают удаление экзосом из кровотока и стимулируют рецепторно-опосредованный эндоцитоз $[6,7]$. Эти белки можно модифицировать методами клик-химии и с помощью создания клеточных культур, экспрессирующих поверхностный белок экзосом с добавленным направляющим пептидом. Направляющий пептид на поверхности экзосом повышает их аффиинность к рецепторам, экспонируемым в целевой ткани [6, 8]. Аналогичным способом к поверхности экзосом можно прикрепить и полезный «груз» - биологические макромолекулы (нуклеиновые кислоты, белки) и низкомолекулярные соединения (ингибиторы, антисмысловые нуклеотиды (АСО)) [6]. Возможность направления экзосом в целевые ткани с помощью модификации их поверхности, низкая иммуногенность этих везикул и высокий потенциал для загрузки могут значительно увеличить их эффективность по сравнению, например, с липосомами, и делают их привлекательными для переноса действующего вещества лекарственных средств. Терапевтические методики, связанные с применением экзосом, находятся в стадии разработки. Чаще всего экзосомы применяют для диагностики: с их помощью, например, достаточно эффективно выявляют онкологические, сердечнососудистые и неврологические патологии [9]. Есть попытки применения экзосом для терапии [9].

\section{Применение экзосом при дистрофии Дюшенна: диагностика}

Для определения наличия дистрофии Дюшенна/Беккера применяют молекулярно-биологические методы (ПЦР, MLPA, секвенирование), которые позволяют определить точную локализацию и тип мутации. Биопсия ткани пациента, применяющаяся в сложных случаях, позволяет определить наличие и локализацию дистрофина и состояние мышечной ткани. Однако контроль за состоянием пациента необходим и в процессе лечения. Неоднократное взятие биопсии не практикуют, так как его может тяжело переносить пациент, к тому же биопсия позволяет оценить состояние только небольшой области одной мышцы. Вместе с тем, функциональный тест 6-минутной ходьбы позволяет оценивать состояние лишь амбулаторных пациентов, а результат выполнения теста зависит от внимательности пациента, его желания и способности следовать методике. Поэтому весьма актуальной представляется задача разработки малоинвазивного и надежного метода для оценки динамики состояния пациента с миодистрофией на протяжении и после терапии. Решением проблемы может оказаться подход, аналогичный жидкостной биопсии (от англ. «liquid biopsy») - анализ экзосомальных микроРНК, полученных из крови пациента. Известен ряд микроРНК (miR-1, miR-21, miR-29, miR-31, miR-29, miR-133, miR133b, miR-206), которые принимают участие в процессах регенерации и дифференцировки мышечной ткани. Три основные «мышечные» микроРHK (miR-1, miR-133 и miR-206) повышаются в сыворотке крови больных МДД, причем повышение уровня miR-206 обнаруживается даже у женщин - носительниц дефектного гена [10]. Есть данные о еще большей группе микроPHK (miR-22, miR-30, miR-95, miR-181, miR-193b, miR-208a, miR-208b, miR-378 и miR-499), которые так же могут послужить маркерами заболевания, однако в этом вопросе требуется подтверждение. Тем не менее, анализ содержания экзосомальных «мышечных» микроРНК во время лечения, например с помощью PMO (phosphorodiamidate morpholino oligomers - морфолиновые олигонуклеотиды, разновидность АСО, используемая для пропуска экзонов, например Eteplirsen [3]), может дать информацию об эффективности терапии [10].

\section{Применение экзосом при дистрофии Дюшенна: терапия нативными экзосомами}

Попытки использовать стволовые клетки для лечения различных патологий предпринимают достаточно давно. В моделях МДД применяют стволовые клетки, полученные из сердечной стромы («клетки, полученные от кардиосфер») [11]. Клинические испытания препарата на основе стволовых клеток сейчас находятся на второй стадии. Основной механизм лечения стволовыми клетками, как считают, обусловлен их способностью стимулировать диффференцировку и регенерацию. По результатам последних исследований, именно экзосомы стволовых клеток являются медиаторами переноса сигнала от стволовых клеток в целевые ткани. Действительно, в исследовании на мышиной модели МДД (мыши mdx) было показано, что интракардиальный [11] и внутривенный [12] способы доставки экзосом, полученных от кардиоссрер, позволили воспроизвести почти все положительные эффекты доставки, осуществляемой самими стволовыми клетами. Интересно отметить, что в мышцах после применения «кардиосферных» экзосом детектировали дистрофин; при этом дистрофин или его мРНК в эКзосомах обнаружены не были. Анализ РНК, содержащихся В «кардиосферных» экзосомах, показал, что они обогащены miR-148a. Внутримышечное введение этой микроРНК привело к частичному восстановлению уровня дистрофина, что указывает на возможный механизм действия «кардиосферных» экзосом [11]. Сходные 
эфффекты (стимуляцию регенерации мышц, снижение степени фиброза и улучшение функционального состояния) вызывают экзосомы, полученные из других источников клеток линии С2C12 [13, 14] и клеток плаценты [15]. В последнем исследовании положительное действие экзосом также обеспечивает доставка микроРНК (в данном случае miR-29). Необходимо отметить, что во всех этих и обсуждаемых ниже работах уделяли мало внимания возможным побочным эффектам введения экзосом. Чаще всего авторы оценивают изменение веса подопытных животных, уровень ферментов печени, азот мочевинь крови, но не рассматривают реакцию иммунной системы.

\section{Применение экзосом при дистрофии Дюшенна: терапия предварительно загруженными экзосомами. Таргетирование экзосом}

Лечение МДД нативными экзосомами представляется перспективным, однако подобный подход в чем-то аналогичен существующим, так как не направлен на решение ключевой проблемы - отсутствия дистрофина Решить эту проблему помогло бы использование экзосом как переносчиков активных веществ. Идея доставлять полноразмерный белок или его ген, или хотя бы редуцированную форму (микродистрофин) выглядит очень привлекательной. Однако длина полноразмерного дистросина составляет примерно 150-180 нм, что превосходит верхнюю оценку диаметра экзосомы. Поэтому, по всей видимости, загрузка в экзосомы белка или кодирующей конструкции будет малоэффективной. Но можно использовать экзосомы для доставки низкомолекулярных соединений. Для лечения МДД применяют синтетические АСО. Так, в одной из работ был отобран пептид, связывающийся избирательно с поверхностным белком экзосом CD63. В дальнейшем конъюгат пептида с АСО обеспечивал более эффективный пропуск экзона по сравнению со свободным АСО. Более того, конъюгат этого пептида с мышечно-специфичным пептидом М12 обеспечивал адресную доставку экзосом в мышцы [6]. В другой работе использовали экзосомы от клеток, трассормированных конструкцией, кодирующей химерный белок Lamp2b (лизосомный белок, экспонирующийся также на поверхности экзосом), слитый с мышечно- или нейроспецифическими пептидами. Модифицированные экзосомы эффективно доставляли эффекторные молекулы (в данном случае это были короткие интерферирующие PHК) в целевые клетки и ткани [8].

\section{ВЫВОДЫ}

Несмотря на большие усилия, эффективное лечение дистрофии Дюшенна по-прежнему не разработано. Существующие методы ограничены, малоэффективны и по большей части симптоматичны. Экзосомы благодаря своей низкой иммуногенности и потенциалу к модификации поверхности могут стать способом доставки действующих веществ. В то же время анализ экзосом пациента может обеспечить диагностику и контроль за прохождением лечения. При этом нельзя не отметить, что методы работы с экзосомами (выделения, стандартизации, загрузки) требуют дальнейшей оптимизации. Следует уделять внимание и иммуногенности экзосом, которая пока недостаточно изучена. Весьма интересной выглядит комбинация обсуждаемых подходов: использование экзосом с регенеративной функцией, загрузка их ACO и модификация поверхностных белков мышечноспецисичными пептидами.

\section{Литература}

1. Birnkrant DJ, Bushby K, Bann CM, Apkon SD, Blackwell A, Brumbaugh D, et al. Diagnosis and management of Duchenne muscular dystrophy, part 1: diagnosis, and neuromuscular, rehabilitation, endocrine, and gastrointestinal and nutritional management. Lancet Neurol. 2018; 17 (3): 251-67. DOI: 10.1016/S1474-4422(18)30024-3. PubMed PMID: 29395989; PMCID: PMC5869704.

2. Duan D. Systemic AAV Micro-dystrophin Gene Therapy for Duchenne Muscular Dystrophy. Mol Ther. 2018; 26 (10): 2337-56. DOI: 10.1016/j.ymthe.2018.07.011. PubMed PMID: 30093306 PMCID: PMC6171037.

3. Lim KR, Maruyama R, Yokota T. Eteplirsen in the treatment of Duchenne muscular dystrophy. Drug Des Devel Ther. 2017; (11): 533-45. DOI: 10.2147/DDDT.S97635. PubMed PMID: 28280301 ; PMCID: PMC5338848.

4. Bunggulawa EJ, Wang W, Yin T, Wang N, Durkan C, Wang Y, et al. Recent advancements in the use of exosomes as drug delivery systems. J Nanobiotechnology. 2018; 16 (1): 81. DOI: 10.1186/ s12951-018-0403-9. PubMed PMID: 30326899; PMCID: PMC6190562.

5. Raposo G, Stoorvogel W. Extracellular vesicles: exosomes, microvesicles, and friends. The Journal of cell biology. 2013 200 (4): 373-83. DOI: 10.1083/jcb.201211138. PubMed PMID: 23420871; PMCID: PMC3575529.

6. Gao X, Ran N, Dong X, Zuo B, Yang R, Zhou Q, Moulton HM, Seow $Y$, Yin $H$. Anchor peptide captures, targets, and loads exosomes of diverse origins for diagnostics and therapy. Sci Transl Med. 2018; 10 (444). DOI: 10.1126/scitransImed.aat0195. PubMed PMID: 29875202
7. Kamerkar S, LeBleu VS, Sugimoto H, Yang S, Ruivo CF, Melo SA, et al. Exosomes facilitate therapeutic targeting of oncogenic KRAS in pancreatic cancer. Nature. 2017; 546 (7659): 498-503. DOI: 10.1038/nature22341. PubMed PMID: 28607485; PMCID: PMC5538883.

8. Alvarez-Erviti L, Seow Y, Yin H, Betts C, Lakhal S, Wood MJ. Delivery of siRNA to the mouse brain by systemic injection of targeted exosomes. Nat Biotechnol. 2011; 29 (4): 341-5. DOI: 10.1038/nbt.1807. PubMed PMID: 21423189.

9. Kim YS, Ahn JS, Kim S, Kim HJ, Kim SH, Kang JS. The potential theragnostic (diagnostic + therapeutic) application of exosomes in diverse biomedical fields. Korean J Physiol Pharmacol. 2018; 22 (2): 113-25. DOI: 10.4196/kjpp.2018.22.2.113. PubMed PMID: 29520164; PMCID: PMC5840070

10. Coenen-Stass AML, Wood MJA, Roberts TC. Biomarker Potential of Extracellular miRNAs in Duchenne Muscular Dystrophy. Trends Mol Med. 2017; 23 (11): 989-1001. DOI: 10.1016/j. molmed.2017.09.002. PubMed PMID: 28988850.

11. Aminzadeh MA, Rogers RG, Fournier $M$, Tobin RE, Guan $X$, Childers MK, et al. Exosome-Mediated Benefits of Cell Therapy in Mouse and Human Models of Duchenne Muscular Dystrophy. Stem Cell Reports. 2018; 10 (3): 942-55. DOI: 10.1016/j. stemcr.2018.01.023. PubMed PMID: 29478899; PMCID: PMC5918344.

12. Rogers RG, Fournier M, Sanchez L, Ibrahim AG, Aminzadeh MA, Lewis $\mathrm{Ml}$, et al. Disease-modifying bioactivity of intravenous cardiosphere-derived cells and exosomes in mdx mice. JCl Insight. 2019; 4 (7). DOI: 10.1172/jci.insight.125754. PubMed PMID: 30944252; PMCID: PMC6483717. 
13. Su X, Shen $Y$, Jin $Y$, Jiang $M$, Weintraub N, Tang $Y$. Purification and Transplantation of Myogenic Progenitor Cell Derived Exosomes to Improve Cardiac Function in Duchenne Muscular Dystrophic Mice. J Vis Exp. 2019; (146). DOI: 10.3791/59320. PubMed PMID: 31033952.

14. Su X, Jin Y, Shen Y, Ju C, Cai J, Liu Y, et al. Exosome-Derived Dystrophin from Allograft Myogenic Progenitors Improves Cardiac Function in Duchenne Muscular Dystrophic Mice. J Cardiovasc

\section{References}

1. Birnkrant DJ, Bushby K, Bann CM, Apkon SD, Blackwell A, Brumbaugh D, et al. Diagnosis and management of Duchenne muscular dystrophy, part 1: diagnosis, and neuromuscular, rehabilitation, endocrine, and gastrointestinal and nutritional management. Lancet Neurol. 2018; 17 (3): 251-67. DOl: 10.1016/S1474-4422(18)30024-3. PubMed PMID: 29395989; PMCID: PMC5869704

2. Duan D. Systemic AAV Micro-dystrophin Gene Therapy for Duchenne Muscular Dystrophy. Mol Ther. 2018; 26 (10): 2337-56. DOI: 10.1016/j.ymthe.2018.07.011. PubMed PMID: 30093306; PMCID: PMC6171037.

3. Lim KR, Maruyama R, Yokota T. Eteplirsen in the treatment of Duchenne muscular dystrophy. Drug Des Devel Ther. 2017; (11): 533-45. DOI: 10.2147/DDDT.S97635. PubMed PMID: 28280301; PMCID: PMC5338848.

4. Bunggulawa EJ, Wang W, Yin T, Wang N, Durkan C, Wang Y, et al. Recent advancements in the use of exosomes as drug delivery systems. J Nanobiotechnology. 2018; 16 (1): 81. DOI: 10.1186/ s12951-018-0403-9. PubMed PMID: 30326899; PMCID: PMC6190562.

5. Raposo G, Stoorvogel W. Extracellular vesicles: exosomes, microvesicles, and friends. The Journal of cell biology. 2013; 200 (4): 373-83. DOI: 10.1083/jcb.201211138. PubMed PMID: 23420871; PMCID: PMC3575529.

6. Gao X, Ran N, Dong X, Zuo B, Yang R, Zhou Q, Moulton HM, Seow $Y$, Yin $H$. Anchor peptide captures, targets, and loads exosomes of diverse origins for diagnostics and therapy. Sci Transl Med. 2018; 10 (444). DOI: 10.1126/scitranslmed.aat0195. PubMed PMID: 29875202.

7. Kamerkar S, LeBleu VS, Sugimoto H, Yang S, Ruivo CF, Melo SA, et al. Exosomes facilitate therapeutic targeting of oncogenic KRAS in pancreatic cancer. Nature. 2017; 546 (7659): 498-503. DOI: 10.1038/nature22341. PubMed PMID: 28607485; PMCID: PMC5538883.

8. Alvarez-Erviti L, Seow Y, Yin H, Betts C, Lakhal S, Wood MJ. Delivery of siRNA to the mouse brain by systemic injection of targeted exosomes. Nat Biotechnol. 2011; 29 (4): 341-5. DOI: 10.1038/nbt.1807. PubMed PMID: 21423189.
Transl Res. 2018; 11 (5): 412-9. DOI: 10.1007/s12265-0189826-9. PubMed PMID: 30155598; PMCID: PMC6212302.

15. Bier A, Berenstein P, Kronfeld N, Morgoulis D, Ziv-Av A, Goldstein H, et al. Placenta-derived mesenchymal stromal cells and their exosomes exert therapeutic effects in Duchenne muscular dystrophy. Biomaterials. 2018; (174): 67-78. DOI: 10.1016/j. biomaterials.2018.04.055. PubMed PMID: 29783118.

9. Kim YS, Ahn JS, Kim S, Kim HJ, Kim SH, Kang JS. The potential theragnostic (diagnostic + therapeutic) application of exosomes in diverse biomedical fields. Korean J Physiol Pharmacol. 2018; 22 (2): 113-25. DOI: 10.4196/kjpp.2018.22.2.113. PubMed PMID: 29520164; PMCID: PMC5840070.

10. Coenen-Stass AML, Wood MJA, Roberts TC. Biomarker Potential of Extracellular miRNAs in Duchenne Muscular Dystrophy. Trends Mol Med. 2017; 23 (11): 989-1001. DOI: 10.1016/j. molmed.2017.09.002. PubMed PMID: 28988850.

11. Aminzadeh MA, Rogers RG, Fournier M, Tobin RE, Guan X, Childers MK, et al. Exosome-Mediated Benefits of Cell Therapy in Mouse and Human Models of Duchenne Muscular Dystrophy. Stem Cell Reports. 2018; 10 (3): 942-55. DOI: 10.1016/j. stemcr.2018.01.023. PubMed PMID: 29478899; PMCID: PMC5918344.

12. Rogers RG, Fournier M, Sanchez L, Ibrahim AG, Aminzadeh MA, Lewis $\mathrm{Ml}$, et al. Disease-modifying bioactivity of intravenous cardiosphere-derived cells and exosomes in $\mathrm{mdx}$ mice. $\mathrm{JCl}$ Insight. 2019; 4 (7). DOI: 10.1172/jci.insight.125754. PubMed PMID: 30944252; PMCID: PMC6483717.

13. Su X, Shen Y, Jin Y, Jiang M, Weintraub N, Tang Y. Purification and Transplantation of Myogenic Progenitor Cell Derived Exosomes to Improve Cardiac Function in Duchenne Muscular Dystrophic Mice. J Vis Exp. 2019; (146). DOI: 10.3791/59320. PubMed PMID: 31033952

14. Su X, Jin Y, Shen Y, Ju C, Cai J, Liu Y, et al. Exosome-Derived Dystrophin from Allograft Myogenic Progenitors Improves Cardiac Function in Duchenne Muscular Dystrophic Mice. J Cardiovasc Transl Res. 2018; 11 (5): 412-9. DOI: 10.1007/s12265-0189826-9. PubMed PMID: 30155598; PMCID: PMC6212302.

15. Bier A, Berenstein P, Kronfeld N, Morgoulis D, Ziv-Av A, Goldstein H, et al. Placenta-derived mesenchymal stromal cells and their exosomes exert therapeutic effects in Duchenne muscular dystrophy. Biomaterials. 2018; (174): 67-78. DOI: 10.1016/j. biomaterials.2018.04.055. PubMed PMID: 29783118. 